\title{
The Dark Energy Survey: cosmological results from the first year of observations
}

\author{
Ramon Miquel*† \\ Institut de Física d'Altes Energies (IFAE), The Barcelona Institute of Science and Technology, \\ Campus UAB, 08193 Bellaterra (Barcelona), Spain. \\ Institució Catalana de Recerca i Estudis Avançats (ICREA), 08010 Barcelona, Spain. \\ E-mail: ramon.miqueleifae.es
}

\begin{abstract}
Since 2013, the Dark Energy Survey (DES) has been mapping an entire octant of the sky to unprecedented depth, measuring the position on the sky, redshift and shape of over 200 million galaxies, together with thousands of galaxy clusters and supernovae. With this data set, DES is studying the properties of dark energy using four main probes: galaxy clustering on large scales, weak gravitational lensing, galaxy-cluster abundance, and supernova distances. The data from the first year of observations (DES-Y1), covering about $1500 \mathrm{deg}^{2}$, have recently been analyzed to derive cosmological constraints from the first three probes, achieving an unprecedented precision in the determination of the cosmological parameters governing the growth of structure in the Universe. Furthermore, the first cosmological results from type-Ia supernovae discovered during the first three seasons of observations (DES-SN3YR) have recently been published. The talk presents and discusses all these results.
\end{abstract}

The 39th International Conference on High Energy Physics (ICHEP2018)

4-11 July, 2018

Seoul, Korea

\footnotetext{
* Speaker.

${ }^{\dagger}$ On behalf of the DES Collaboration.
} 
The Dark Energy Survey (DES $\left.{ }^{1},[1]\right)$ is an on-going, six-year survey that, when completed, will map over 200 million galaxies and tens of thousands of galaxy clusters in five filters ( $\mathrm{griz} Y$ ) over $5000 \mathrm{deg}^{2}$, in addition to discovering several thousand type-Ia supernovae in a $27 \mathrm{deg}^{2}$ timedomain survey.

DES will use several cosmological probes to test the standard $\Lambda$ CDM model of cosmology; galaxy clustering and weak gravitational lensing are two of the most powerful. Jointly, these complementary probes sample the underlying matter density field through the galaxy population and the distortion of light due to gravitational lensing, so that one can determine simultaneoously the cosmological parameters and the galaxy bias. The joint analysis of galaxy clustering, galaxygalaxy-lensing and cosmic shear (dubbed " $3 \times 2$ analysis") in the DES first-year data (DES-Y1) was presented in a series of papers that culminated in [2] (see also references therein), from where Fig. 1 is extracted. The plot presents the measurement of what amounts to the first two central

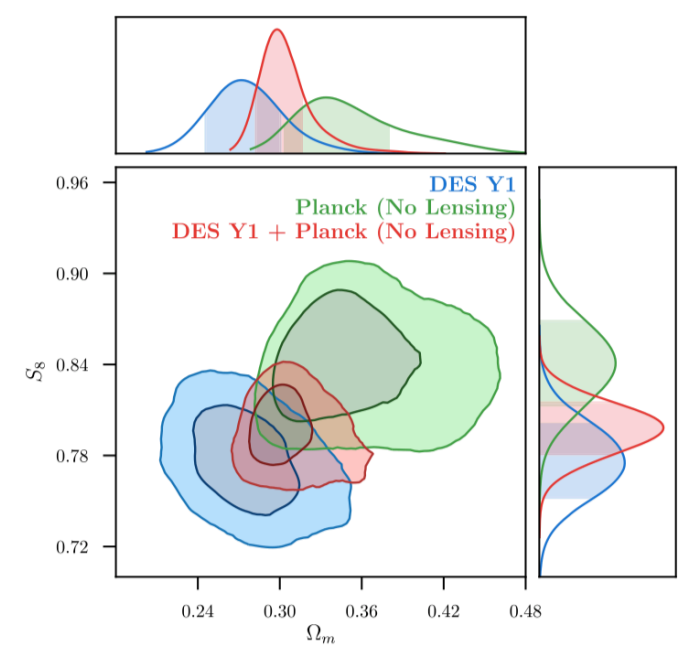

Figure 1: $\Lambda \mathrm{CDM}$ constraints from the combination of galaxy clustering and weak lensing in DES Y1 (blue), Planck with no lensing [3] (green), and their combination (red). A Bayesian analysis establishes that in the full multidimensional paramenter space the two data sets are consistent.

moments of the (mostly dark) matter distribution in the late universe: $\Omega_{M}$ is the mean matter density now in units of the critical density and $S_{8}=\sigma_{8} \sqrt{\Omega_{m} / 0.3}$, where $\sigma_{8}$ is the RMS of the matter density measured in spheres of radius $8 \mathrm{Mpc} / \mathrm{h}$. This is the first such measurement that achieves a precision that matches that obtained by extrapolating to redshift zero the measurement of the anisotropies of the CMB at redshift 1100 assuming the $\Lambda$ CDM model. As such, the agreement between the Planck and DES measurements constitutes a test of $\Lambda$ CDM.

The galaxy distribution in the sky presents a feature at separations $\simeq 100 \mathrm{Mpc} / \mathrm{h}$ that acts as a standard ruler of known length, calibrated by the CMB. By measuring it at different redshifts, one can test the underlying cosmological model. This measurement has been performed with the DESY1 data set in a redshift region previously unexplored [4]. The result is shown in Fig. 2, where it is compared to other results also measuring the angular BAO feature (in the transverse direction), and not along the line of sight (which is impossible for DES, given the limited redshift resolution).

\footnotetext{
${ }^{1}$ http: / / www. darkenergysurvey.org
} 


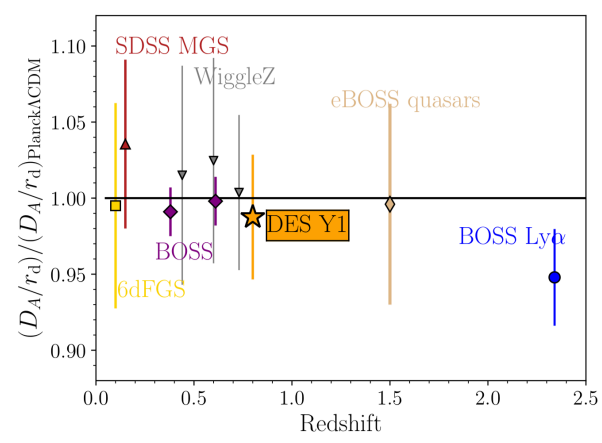

Figure 2: Measurement of the angular diameter distance obtained from BAO, compared to the Planck $\Lambda \mathrm{CDM}$ prediction. The DES Y1 measurement is shown using a gold star. For experiments that measured spherically averaged distances, we have multiplied the uncertainty by 1.5 .

The results agree with the prediction from the $\Lambda$ CDM model. This is the highest-redshift BAO measurement from an imaging survey.

Another very sensitive probe of dark energy is the distribution of clusters in redshift and mass. Here, the tricky part is to calibrate the indirect measurements of the cluster mass, based, for instance, in their richness. DES has shown that this calibration can be achieved at the 5\% level using weak-lensing measurements [5]. The cosmological results using a sample of 6500 clusters in the redshift range $0.2<z<0.65$ from the DES-Y1 data set are undergoing internal review within DES. For the moment, we can show in Fig. 3 the blinded results in the $\Omega_{m}-\sigma_{8}$ plane. The sensitivity of

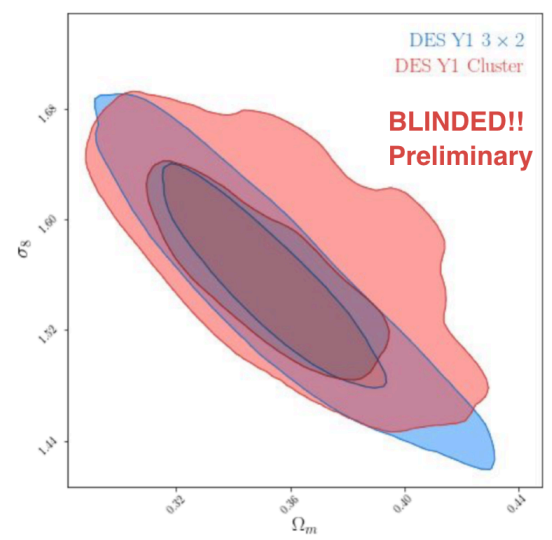

Figure 3: Blinded preliminary results from the DES-Y1 analysis of galaxy cluster abundance as a function of mass and redshift (red). The contours are centered on the result obtained from the galaxy clustering plus lensing $(3 \times 2)$ analysis (blue). One can see that the expected accuracy from galaxy clusters almost matches that from the $3 \times 2$ analysis.

the method is comparable to the one obtained from the combination of galaxy clustering and weak lensing. 
Finally, DES has recently published [6] its first cosmological results from supernovae, based on a sub-sample of 207 spectroscopically confirmed type-Ia supernovae up to $z<0.85$ discovered in the first three years of the survey (DES-SN3YR), for which distances have been measured with a $4 \%$ accuracy. Figure 4 shows the contours obtained in the $\Omega_{M}-w$ plane, where $w$ is the equation of state of the dark energy, assumed constant. The figure shows how the combination of the DES SNe

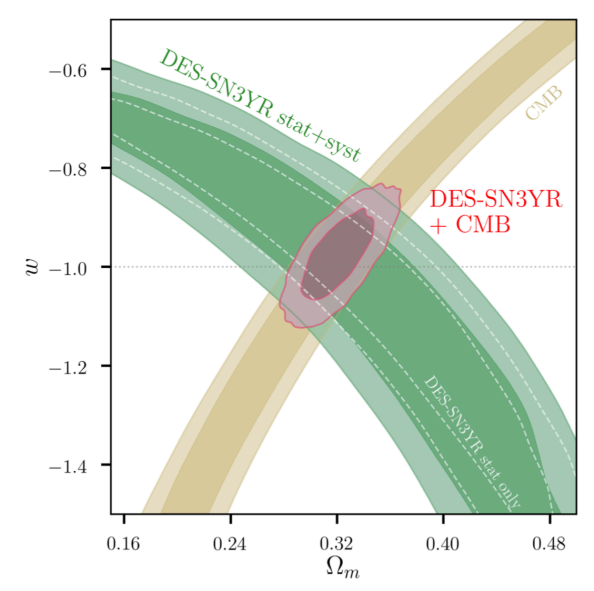

Figure 4: Constraints on $\Omega_{m}-w$ for a flat wCDM model. SN contours are shown with only statistical uncertainty (white-dashed) and with total uncertainty (green-shaded). Constraints from CMB (brown) and DES-SN3YR+CMB combined (red) are also shown.

results with the CMB results from Planck produces tight constraints on $w$, which appears consistent with a cosmological constant $\Lambda(w=-1)$.

In summary, analyzing its first year data set DES has produced cosmological results from the four main probes of dark energy, achieving unprecedented precision. In 2019, the combination of the results from all probes in the 3-year data set will provide very stringent constraints on the standard cosmological model.

\section{References}

[1] DES Collaboration, arXiv:astro-ph/0510346.

[2] DES Collaboration, PRD 98 (2018) 043526.

[3] Planck Collaboration, A\&A 594 (2016) A13.

[4] DES Collaboration, arXiv:1712.06209 [astro-ph.CO].

[5] T. McClintock et al. (DES Collaboration), arXiv:1805.00039 [astro-ph.CO].

[6] DES Collaboration, arXiv:1811.02374 [astro-ph.CO]. 